is important to take pre-abortion mental health into account (which we did).

Rowlands \& Guthrie also suggest that an adequate review of this issue has been conducted by Charles et al. ${ }^{3}$ We do not agree. This review has been criticised on the grounds of investigator bias ${ }^{4}$ and these concerns are supported by the somewhat anomalous conclusions the authors draw. For example, the study by Schmierge \& Russo $^{5}$ using the National Longitudinal Study of Youth (NLSY) data is ranked as one of the four 'good' studies despite the facts that only $40 \%$ of women having an abortion were included, and mental health was measured using a single scale of depression, with this outcome being assessed up to 13 years after the abortion. All of these features will bias results towards the null hypothesis. In addition, Charles et al ${ }^{3}$ failed to distinguish between the different causal questions that may be asked about abortion, and misleadingly dismissed a number of studies showing links between abortion and mental health on the grounds that they did not include an appropriate comparison group.

Finally, Rowlands \& Guthrie claim that conclusions cannot be drawn about the causal effects of abortion on mental health in the absence of randomised controlled trials. We believe that this argument overstates the case and there is now growing evidence for two major conclusions about abortion and mental health. First, exposure to abortion is an adverse life event which is associated with a modest increase in risks of mental health problems. Second, the mental health risks associated with abortion may be larger, and certainly are not smaller, than the mental health risks associated with unwanted pregnancies that come to term.

1 Fergusson DM, Horwood $\mathrm{L}$, Boden JM. Abortion and mental health disorders: Evidence from a 30 year longitudinal study. Br J Psychiatry 2008; 193 : 444-51.

2 Dingle K, Alati R, Clavarino A, Najman JM, Williams GM. Pregnancy loss and psychiatric disorders in young women: an Australian birth cohort study. Br J Psychiatry 2008; 193: 455-60.

3 Charles VE, Polis CB, Sridhara SK, Blum RW. Abortion and long-term mental health outcomes: a systematic review of the evidence. Contraception 2008; 78: $436-50$

4 Ertelt S. Researcher: Johns Hopkins paper biased on abortion-mental health issues. LifeNews.com, 2008; 4 December (http://www.lifenews.com/ nat4629.html).

5 Schmiege S, Russo NF. Depression and unwanted first pregnancy: longitudinal cohort study. BMJ 2005; 331: 1303.

David M. Fergusson, Christchurch Health and Development Study, University of Otago, Christchurch School of Medicine and Health Sciences, PO Box 4345, of Otago, Christchurch School of Medicine and Health Sciences, PO Box 4345,
Christchurch, New Zealand. Email: dm.fergusson@otago.ac.nz; L. John Horwood, Joseph M. Boden, Christchurch Health and Development Study, University of Otago, Christchurch School of Medicine and Health Sciences, Christchurch, New Zealand

doi: 10.1192/bjp.195.1.83a

\section{Longitudinal course of cognition in schizophrenia}

In their meta-analysis, ${ }^{1}$ Szöke et al found significant improvements in most neuropsychological variables, along with well-known potential practice effects, and that semantic verbal fluency holds promise as a suitable cognitive endophenotype in schizophrenia. We acknowledge that this review is a step forward, attempting to merge and quantify the evidence from both naturalistic observational studies ${ }^{2}$ and clinical trials. ${ }^{3}$

We agree with the authors that the current literature is limited by the virtual absence of healthy control groups. Since only 9 out of 53 studies reported longitudinal neurocognitive data for controls, it is difficult to disentangle whether patients' cognitive changes are true improvements or confounded by the non-specific effects of practice-related learning. ${ }^{4}$ The use of healthy individuals from test-retest studies ('external controls') is also problematic and the parallel assessment of controls would rule out the possibility that patients' apparent cognitive stability is not deterioration in disguise, as the authors accurately suggest.

In addition, we would like to highlight other critical issues that may limit the conclusions. First, the authors' choice to lower the minimum study duration to 1 month led to the median test-retest interval being only 4 months, which is shorter than the recommended trial duration to evaluate cognitive changes (e.g. at least 6 months). ${ }^{5}$ This also may have biased the review towards short-term clinical trials intended to improve cognitive deficits, especially with second-generation antipsychotics. Had a more stringent and clinically relevant 1-year follow-up cut-off ${ }^{2}$ been used, only 24 out of 53 studies would have been reviewed. Second, approximately half of the 20 clinical trials previously reviewed ${ }^{3}$ are open, and these are mostly clozapine trials of patients who are treatment-resistant. Significant associations between cognitive change and change in negative symptoms are more likely to occur in these studies than in naturalistic follow-up studies. ${ }^{5}$ Third, we feel the authors missed an opportunity to include the distinction between patients with first-episode or chronic schizophrenia and geriatric patients as a potential moderator variable. This could have contributed to a better understanding of the probably complex cognitive pathways during the lifespan.

Despite the number of longitudinal neurocognitive reports, less is known than was originally supposed about the course of cognition in schizophrenia. Only a small subset $(n=4)$ of longitudinal reports have compared these neurocognitive pathways with those of healthy controls over at least 1 year. When reviews ${ }^{2}$ are not focused on the neurocognitive effects of antipsychotics, stable long-term performances and, in some cases, cognitive gains could be expected, thus offering a rather pessimistic picture for cognitive enhancement. This approach seems more useful for understanding the long-term natural history of cognition in schizophrenia. Conversely, this meta-analysis ${ }^{1}$ relies on short-term data, mostly from clinical trials, ${ }^{3}$ and therefore more likely overestimates the potential for cognitive improvement.

In summary, it would be misleading if the conclusions were regarded as a major leap forward instead of as tentative hypotheses awaiting further investigation. Since the possibility remains that the current findings are more valuable to researchers, a new metaanalysis that takes into consideration these and other limitations might be more helpful for clinicians, patients and caregivers.

1 Szöke A, Trandafir A, Dupont ME, Méary A, Schürhoff F, Leboyer M. Longitudinal studies of cognition in schizophrenia: meta-analysis. Br J Psychiatry 2008; 192: 248-57.

2 Rund BR. A review of longitudinal studies of cognitive functions in schizophrenia patients. Schizophr Bull 1998; 24: 425-35.

3 Woodward ND, Purdon SE, Meltzer HY, Zald DH. A meta-analysis of neuropsychological change to clozapine, olanzapine, quetiapine, and risperidone in schizophrenia. Int J Neuropsychopharmacol 2005; 8: 457-72.

4 Goldberg TE, Goldman RS, Burdick KE, Malhotra AK, Lencz T, Patel RC, et al. Cognitive improvement after treatment with second generation antipsychotic medications in first-episode schizophrenia: is it a practice effect? Arch Gen Psychiatry 2007; 64: 1115-22.

5 Buchanan RW, Davis M, Goff D, Green MF, Keefe RS, Leon AC, et al. A summary of the FDA-NIMH-MATRICS workshop on clinical trial design for neurocognitive drugs for schizophrenia. Schizophr Bull 2005; 31: 5-19.

Vicent Balanzá-Martínez, Department of Medicine, University of Valencia, Blasco Ibáñez, 15, 46010 Valencia, Spain; Email: vicente.balanza@uv.es; Manuel J. Cuesta Psychiatric Unit, Virgen del Camino Hospital, Pamplona; Celso Arango, Psychiatry Department, Adolescent Unit, University Hospital Gregorio Marañón, CIBERSAM, Madrid; Benedicto Crespo-Facorro, University Hospital Marqués de Valdecilla, Department of Psychiatry, School of Medicine, University of Cantabria, CIBERSAM, Santander; Rafael Tabarés-Seisdedos, Section of Psychiatry and Psychological Medicine, Department of Medicine, University of Valencia, CIBERSAM, Valencia, Spain

doi: 10.1192/bjp.195.1.84 